\title{
Prevalence of Oral Lesions in Dermatological Diseases: A Retrospective Study
}

Thays Teixeira de Souza, ${ }^{1}$ Thiago Moreira Pêssoa, ${ }^{1}$ Ruth Tramontani Ramos, ${ }^{1}$ Marilia Heffer Cantisano, ${ }^{1}$ Geraldo Oliveira Silva-Júnior ${ }^{1}$

${ }^{1}$ Department of Diagnosis and Therapeutics, School of Dentistry, Rio de Janeiro State University, Rio de Janeiro, RJ, Brazil

- Conflicts of interest: none declared.

\section{Abstract}

Objective: this study aimed to establish the prevalence of oral manifestations in dermatological diseases. Material and Methods: the study population consisted of 119 patients with skin diseases (according to medical records). Demographic and clinical data collected include sex, skin color, age of diagnosis, and anatomical location of lesion. Results: there was a significant prevalence of females $(n=74 ; 63 \% ; p=0.01)$ and white skin color $(n=7 ; 65 \% ; p<0.001)$. The age of diagnosis ranged from 10 to 86 years, with a high prevalence of individuals $>20$ years old $(n=86 ; 73 \% ; p=0.05)$. The most frequent lesion was pemphigus vulgaris (38\%) followed by oral lichen planus (30\%); Mucous Membrane Pemphigoid (17\%); Lupus Erythematosus (10\%); Erythema Multiforme and Epidermolysis Bullosa (3\%); Chronic Ulcerative Stomatitis and Pyostomatitis Vegetans (1\%). Conclusion: dermatological diseases may affect the oral mucosa, causing a significant impact on the quality of life of the patients, which mainly comprised the young populace in our study. Pemphigus vulgaris was the most prevalent disease. Continuous monitoring by a multidisciplinary team is essential, since this group of disorders has no cure and/or variable prognosis.

Keywords: Dermatological diseases; Oral lesions, Lichen planus; Pemphigus vulgaris; Pemphigoid; Erythema multiforme; Systemic lupus erythematosus; Chronic ulcerative stomatitis; Pyostomatitis vegetans; Epidermolysis bullosa

\section{Introduction}

$\mathrm{T}$ There is increasing evidence on the steady rise of dermatological diseases in the last decades. ${ }^{1}$

Several of these conditions are caused by the inadequate production of antibodies against the causal organism of these diseases. ${ }^{1}$ In this context, some of these diseases are physically expressed such as lichen planus, pemphigus vulgaris, pemphigoid, systemic lupus erythematosus, erythema multiforme and chronic ulcerative stomatitis, ${ }^{2}$ with the oral manifestations frequently being the primary sign of these conditions. ${ }^{3}$

Pemphigus diseases are rare immune-mediated bul $\neg$ lous diseases, which affects the skin and mucous membranes that break through minor trauma, culminating in painful ulcers and susceptibility to infection. Nearly all patients present signs of mucosal lesions, mainly in the oral mucosa, with or without cutaneous lesions. ${ }^{4}$

Oral lichen planus (OLP) is a chronic inflammatory disease of the skin and mucous membranes, which frequently manifests in the oral mucosa before or after epidermal appearances. These lesions are characterized by Wickham's stretch marks and various clinical presentations, which are mainly reticular and erosive. ${ }^{5,6}$

Mucous membrane pemphigoid (MMP) is a group of immune-mediated chronic blistering conditions. It targets the oral mucosa as well as the genitals, skin, and particularly, conjunctival and mucous membranes. ${ }^{7}$ Compared to pemphigus, pemphigoid blisters are less brittle and remain intact in the oral cavity for up to 48 hours. ${ }^{8}$
Erythematosus lupus is an autoimmune disease classified as either systemic lupus erythematosus (SLE) or cutaneous lupus erythematosus (CLE). ${ }^{8,9}$ Oral involvement ranges from $5 \%-54 \%$ in systemic lupus erythematosus (SLE) and $3 \%-25 \%$ in cutaneous lupus erythematosus (CLE). ${ }^{9}$

Erythema multiforme (EM) is an acute, self-limiting, immune-mediated disorder that affects the skin and/or mucosal surfaces. The most frequent site involved is the oral mucosa, with a prevalence of up to $70 \% .^{10}$

Chronic Ulcerative Stomatitis (CUS) is a rare chronic condition that causes painful, exacerbat $\neg$ ing, and remitting ulcerations, particularly in oral mu $\neg$ cous membranes. ${ }^{11}$

Other conditions [such as pyostomatitis vegetans (PYV)] are characterized as inflammatory and eosinophilic conditions which affects the skin as well as oral, genital and conjunctural mucous membranes. These conditions are greatly influenced by gastrointestinal inflammatory disease. In the oral mucosa, lesions are characterized mainly by multiple vesicles that appear under the erythematous base, which are usually ruptured, thus manifesting as ulcer. ${ }^{12}$

Rare dermatological conditions occur due to genetic alterations different from the above described conditions such as epidermolysis bullosa (EB). EB can be classified into many clinical subtypes. It is a heterogeneous disease with a broad spectrum of severity. Oral, esophageal, tracheal, genitourinary, and ocular mucosal membranes may be affected by erosions, ulcerations, and scarring. Progressive scarring results in contractures and/or mutilations of extremities, microstomia, disfigurement 
and esophageal stenosis. ${ }^{13}$

It is important to emphasize that oral lesions can have a huge impact on quality of life of patients since they inflict pain, causing functional, and social limitations.

Dentists can play a pivotal role in detection and treatment of these conditions. Moreover, precise and early diagnosis increases the efficiency and efficacy of treatment strategy. ${ }^{14}$

This study aimed to establish the prevalence of oral manifestations in dermatological diseases; their relationship with severity of the disease, and consequent impact on patient's quality of life.

\section{Material and Methods}

The sample consisted of 119 patients with skin diseases (according to medical records from Stomatology Service of the Piquet Carneiro Polyclinic-State University of Rio de Janeiro) treated between 2009 and 2019. All patients presented clinical and histopathological signs of some immune-mediated, inflammatory or hereditary skin disease. As an inclusion criterion, we considered patients whose medical records had all legible information needed for the study such as definitive histopathology findings and well-maintained complementary findings. As an exclusion criterion, we considered descriptive histopathological findings, incomplete medical records without final followup of patient, as well as outdated and poorly maintained complementary findings. Demographic and clinical data including sex, skin color, age of patient at diagnosis, and anatomical location of lesion were collected from medical records. Before any procedure, authorization was requested from patients or persons responsible for signing the consent form. The study was approved by the Research Ethics Committee (protocol No. 2540 / 0078.0228.000-09).

Data were collated in a database (Microsoft Office Excel 2013) for analysis. Statistical descriptions of variables were done using proportions (that is, for categorical variables) and the median, standard deviation, and minimum and maximum values were calculated (that is, for quantitative variables). Pearson's Chi-Square Test $\left(x^{2}\right)$ and Fisher's Exact Test were applied using Graph Pad Prism Version 6.07 Windows software (Graphpad, San Diego, California, USA) for analysis of the associations between oral lesions and their clinical and demographic characteristics at a p-value of 0.05 and confidence interval of $95 \%$.

\section{Results}

Of the total 119 cases, there was a significant prevalence of females $(\mathrm{n}=74 ; 63 \% ; \mathrm{p}=0.01)$ and white skin color $(\mathrm{n}=$ $7 ; 65 \% ; \mathrm{p}<0.001)$. The age of diagnosis was of the ranged of
10-86 years, with the highest prevalence among individuals aged $>20$ years $(73 \% ; p=0.05)$. The most frequent lesion was pemphigus vulgaris (38\%). The most common site was the cheek mucosa $(38 \%$; $<0.0001)$ followed by tongue and gingiva (Table 1).

Pemphigus vulgaris was observed in 43 patients, with higher prevalence among females (53\%), individuals $>20$ years of age; and predominant involvement of tongue, cheek mucosa, and gingiva (which is mainly in white skin). As for painful complaints, $93 \%$ of the patients were asymptomatic (Table 1).

OLP was the second most commonly found lesion in 36 patients, with higher prevalence among females (69\%), individuals $>20$ years of age, and white skin color (50\%). In addition, the lesions had greater distributions in the tongue, cheek mucosa and gingiva. $78 \%$ of the patients who were asked about the presence of symptoms gave a positive response (Table 1).

The third most common lesion was MMP (17\%), with predominance among females (53\%) and leucoderms (84\%). $100 \%$ of the cases were individuals $>20$ years of age; and the most prevalent distribution site was the tongue, cheek mucosa and gingiva, with $89 \%$ of the patients experiencing pain (Table 1).

The cases of lupus erythematosus was 11 (10\%), with significant prevalence among females (91\%) and white skin color $(82 \%)$. The lesion sites varied with a simultaneous predominance in the tongue, cheek mucosa and gingiva (36\%), in which $73 \%$ of the patients had pain (Table 1 ).

Patients with EM was low, comprising only 4 cases. The age distribution at diagnosis was $50 \%$ for patients aged $\leq 19$ years and $50 \%$ for patients aged $\geq 20$ years. The sites of lesions was cheek mucosa, tongue and gingiva, simultaneously; and on the lips, with 2 lesions on each side. All cases were symptomatic (Table 1).

Only 4 patients had EB, with $100 \%$ white skin color, $75 \%$ females; and lesions sites at the tongue, cheek mucosa and gingiva, simultaneously, with painful symptoms. The age distribution at diagnosis was $75 \%$ for patients aged $\leq 19$ years and $25 \%$ for patients aged $\geq 20$ years old.

We found only one case of CUS, which affected a 63-year-old white woman, with lesions located on the tongue, cheek mucosa and gingiva, asymptomatically (Table 1).

Similarly, only 1 case of pyostomatitis vegetans was obtained, which affected a black man over 20 years of age, with lesions on the tongue, cheek mucosa and gingiva, simultaneously, without painful symptoms.

The clinical lesions of each group of diseases are shown in Figure 1. 


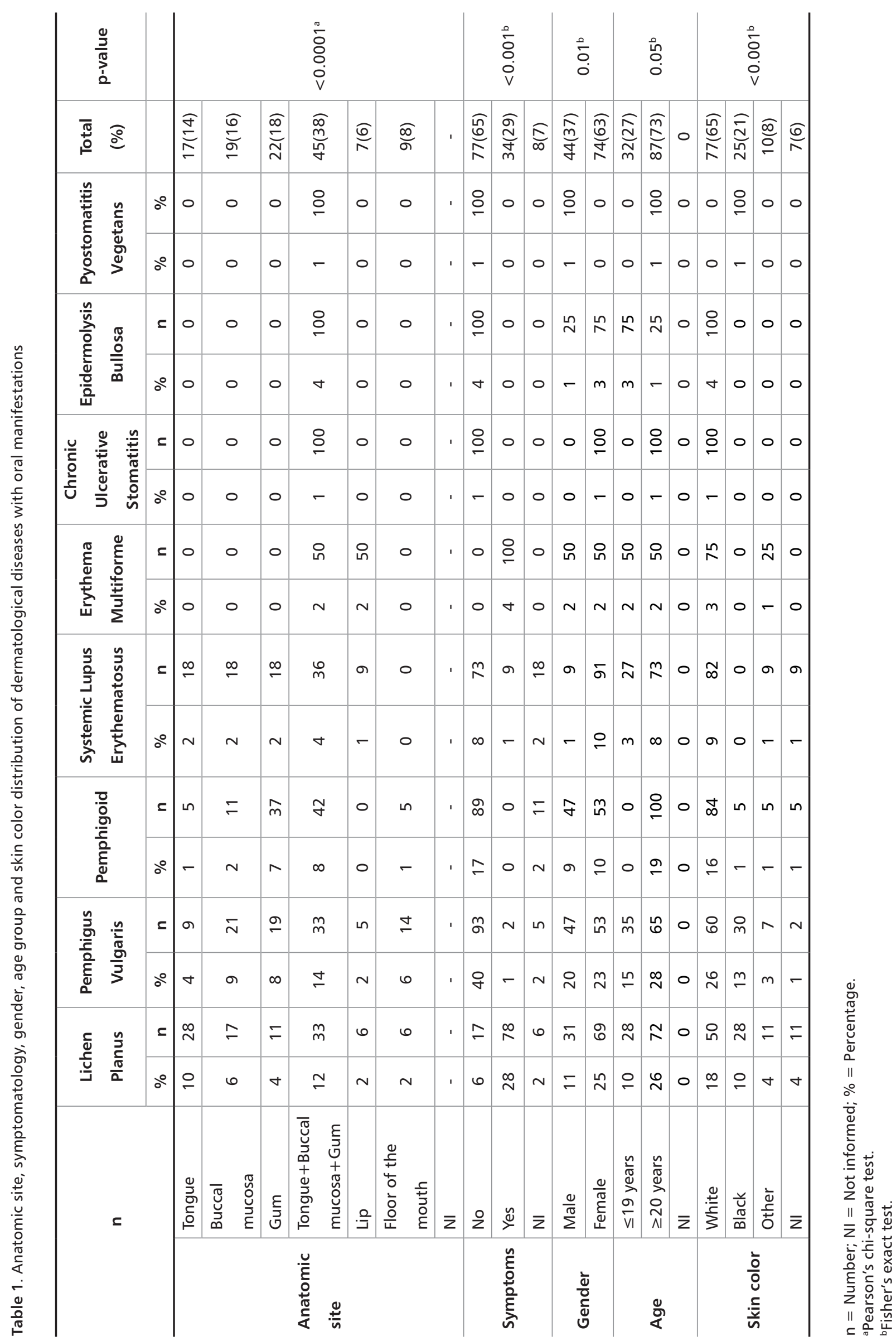



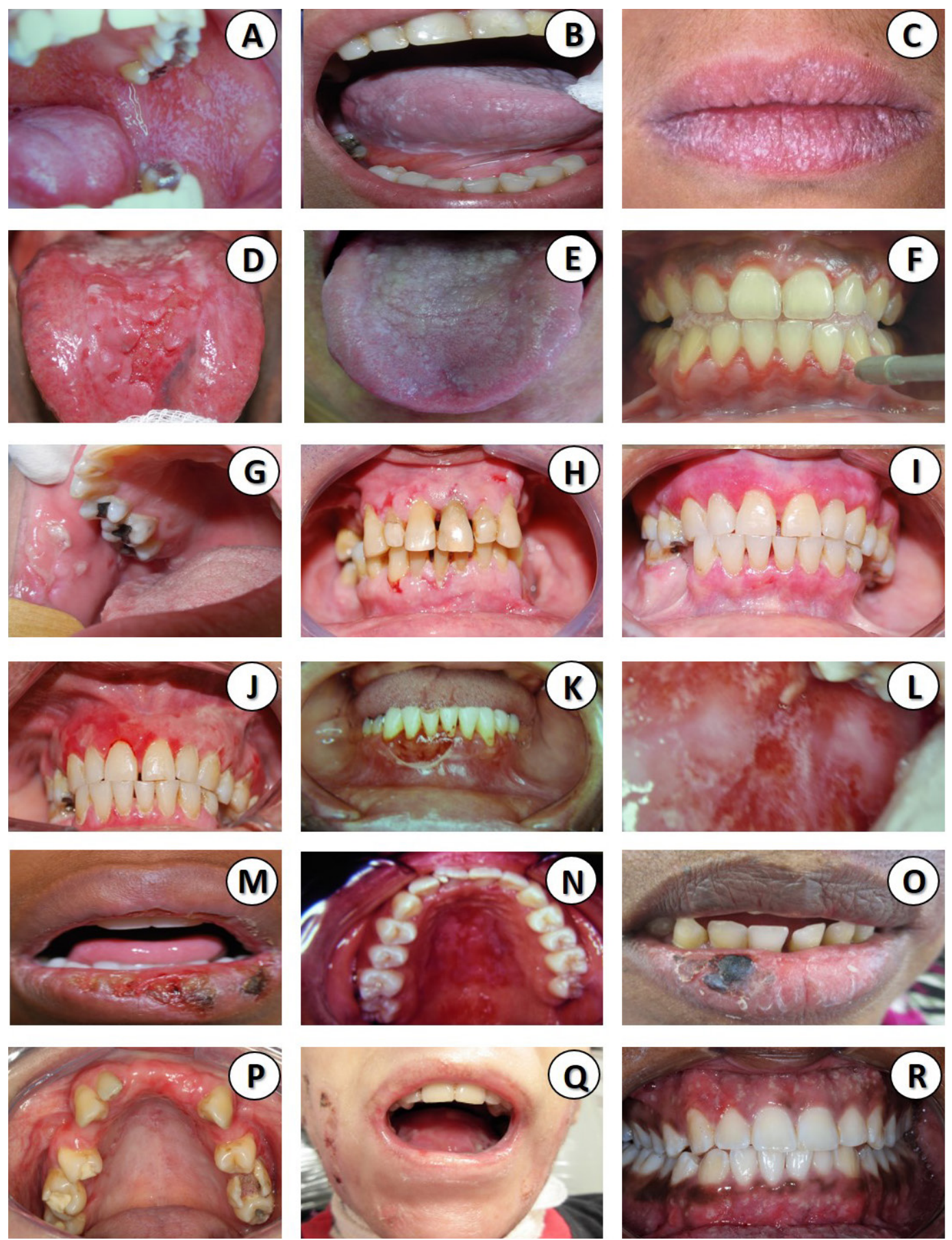

Figure 1. Oral clinical characteristics of the main dermatological diseases. A. Oral Liquen Planus-Reticular, B. Oral Liquen Planus-plaques, C. Oral Liquen Planus-forma reticular, D. Oral Liquen Planus erosive/bullous, E. Oral Lique Planus-forma papular, F. Pemphigus Vulgaris-with desquamative gengivitis and positive Nikolsky signal, G. Pemphigus Vulgaris, H. Pemphigus Vulgaris, I. Mucous Membrane Pemphigoid, J. Mucous Membrane Pemphigoid - positive Nikolsky signal, K. Mucous Membrane Pemphigoid, L. Mucous Membrane Pemphigoid, M. Erithema Multiforme, N. Systemic Lupus Erythematosus, O. Systemic Lupus Erythematosus, P. Chronic Ulcerative Stomatitis, Q. Recessive Dystrophic Epidermoysis Bullosa-Microstomy, R. Pyoestomatitis vegetans

\section{Discussion}

Dermatological diseases include a large group of diseases with important oral manifestations, which often require proper diagnosis and appropriate referral for systemic investigation and treatment of the disease. The sample represented $4.18 \%$ of the cases out of the 2850 patients medical record consulted, indicating a relatively low index of the diseases that affect the oral cavity with initial manifestations. According to our results (Table 1), the prevalence of dermatological lesions with oral manifestations may be different in the same country. This emphasizes that multicentre studies contribute to the understanding of epidemiological differences in population, thus better characterizing the heterogeneity of dermatomucous orifices 
in extensive territories such as Brazil and even globally.

When the disease behavior was assessed based on oral cavity, gender, age and skin color, the differences were statistically significant for each parameter analyzed, specifically in terms of anatomical location of lesions ( $\mathrm{p}<$ $0.0001)$, presence of symptoms $(\mathrm{p}<0.001)$ and skin color $(\mathrm{p}$ $<0.001$ ), obtaining more statistically significant differences when correlated with the diseases (Table 1).

Pemphigus vulgaris is a chronic autoimmune vesiculobullous disease directed against desmossomal proteins, which occur at epithelial junctions of the lining tissues, especially desmoglein 1 and $3 .^{14,15}$

Oral lesions are seen in almost all patients in association (or not) with skin lesions. They can be initially observed as symptomatic oral lesions in $50 \%-70 \%$ of cases and with $90 \%$ of patients without the disease. ${ }^{16}$

In this study, patients affected by PV were mostly females, a fact that is reaffirmed in literature which shows a male/female prevalence of 1:1.5 up to 1:9.17-18 The location of the lesions is the same as described by other the authors 18 who reported high prevalence in the cheek mucosa, gingiva, and palate. It is necessary to emphasize that in our study, $33 \%$ of the cases simultaneously presented more than one affected area. Few cases were reported in literature to affect children and young people, ${ }^{18}$ suggesting that this disease manifests mostly in adults. However, an increase in the frequency of PV has been observed in younger patients. These numbers seem to be higher in the Middle East and Brazil,${ }^{17-19}$ which according to a study was $17.7 \%$ cases in individuals aged $<30$ years. ${ }^{17}$ In our study, $35 \%$ of the affected patients were diagnosed before the age of 19 years, corroborating aforementioned data in literature. Most studies suggest that anxiety, stress, and depression may act as environmental factors in the etiopathogenesis of PV in recent decades and that these cases may occur at any age, as well as in children and adolescents. ${ }^{19}$

Some studies have demonstrated a familial and hereditary pattern in the etiopathogenesis of PV in association with HLA (Human Leukocyte Antigen) genes. ${ }^{20}$ Analysis were with HLA-DRB1 ${ }^{\star} 0402 / 1401 / 1404, \mathrm{DQB1}^{*} 0503$ and $\mathrm{DQB1}^{*} 03 / 07 / 15$ in the most diverse populations. ${ }^{20}$ With the advent of molecular methods and genetic mapping of increasingly easy and fast execution, diagnoses can be obtained with greater precision and precocity, which also justifies the increasing number of young individuals diagnosed with immunemediated disorders such as PV.

Treatment for PV involves regulation of immune dysfunction with corticosteroids and immunosuppressants. In oral lesions, topical corticosteroids can be applied along with systemic treatments; however, the concomitant use of biomodulation through low-level laser therapy has been recently shown to regulate pain and promote analgesia, in addition to the significant healing benefit. ${ }^{21}$
OLP is another chronic inflammatory mucocutaneous disorder that has an unknown origin. However, it is suggested to be mediated immunologically by T cells. ${ }^{18,22}$ Gupta \& Jawanda (2015), in their review, estimated that the prevalence of this disorder is 0.5 to $2.0 \%$ in the general population, with greater prevalence in females and white skin, ${ }^{22}$ which is in agreement with our study.

Mucocutaneous disorder have been reported to have the highest rate of oral lesions; ${ }^{22}$ however, we observed a greater number of pemphigus vulgaris cases in our study. It is possible that cases diagnosed as OLP described in literature are Oral Lichenoid Reactions (OLR), considering the extreme clinical and histopathological similarity, which makes definitive diagnosis difficult. Werneck et al (2016) highlighted the importance of evaluating and treating the association of OLP lesions with candidiasis due to changes in inflammatory infiltrate pattern, which facilitates differential histopathological diagnosis of OLR, ${ }^{23}$ a principle adopted in our stomatology sector through clinical and cytopathological examinations. Other measures for diagnostic confirmation such as use of direct immunofluorescence can also be applied. Depending on the clinical aspect of OLP lesions and severity of the disease, histopathological examination alone may be insufficient. ${ }^{22}$ This differentiation is particularly useful in cases of desquamative gingivitis, in which the clinical aspects of OLP are practically indistinguishable from other vesiculobullous diseases such as pemphigus vulgaris and mucous membranes pemphigoid. ${ }^{24}$

Like pemphigus vulgaris, many cases of lichen planus are triggered by psychological stress. Moreover, acute exacerbations seem to be directly linked to depression and anxiety, both of which are factors found to increase the frequency in younger individuals, which suggestively accounts for the percentage found in patients under 19 years old $(28 \%)$ in our study. ${ }^{25}$

Being a disease without cure, treatment consists of the relief and improvement of symptoms as presented in $78 \%$ of patients with OLP in our study. The clinical presentations are quite varied, ranging from white streaked areas and ulcers and erosions. In 33\% of patients in our study, lesions were diffusely distributed in the oral mucosa, showing greater severity in the manifestations of OLP and possibly greater resistance to the proposed treatment. Local factors are also directly linked to the disease exacerbation such as poorly adapted prostheses, unsatisfactory restorations, and periodontal disease with large accumulations of plaque. ${ }^{25}$

Basically, corticotherapy is the first line of treatment for symptomatic cases with ulcerated and/or erosive lesions. Topical agents are preferred to systemic agents because they minimize side effects; however, their combination can also be effective. ${ }^{26}$ In the absence of response to these therapeutic agents, immunosuppressants such as cyclosporine and 
azathioprine are effective options. ${ }^{27}$

Recently, some alternative therapies such as medicinal herbs and ozone therapy have been proposed. However, further scientific analysis is required to validate thier efficacy. ${ }^{28,29}$

MMP, like pemphigus vulgaris, are vesiculobullous diseases, which are autoimmune, inflammatory, and chronic diseases characterized by formation of subepithelial bubbles and linear deposition of $\operatorname{IgG}, \operatorname{Ig} \mathrm{A}$, or C3 along the basement membrane area. Lesions usually affect oral, conjunctival, genital, and digestive mucosa; however, skin manifestations are rare. The oral mucosa is the most involved site, with $85 \%$ of cases. ${ }^{30}$

Clinically, it manifest as areas of erosion and/or ulcerations throughout the mucosa, with gingiva being the predominantly affected site, which is recognized as desquamative gingivitis. However, this term does not refer only to MMP lesions, but may also be present in cases of OLP and PV. ${ }^{14}$ Similar to the literature, most of the patients in our study presented lesions in the gingiva (both isolated and diffused) followed by cheek mucosa and tongue.

MMP generally occurs in patients between their 5th and 6th decades of life, thus corroborating our study in which $100 \%$ of cases were diagnosed among older cohorts. Nevertheless, it also affect patients at their earlier ages. ${ }^{30,31}$ It is believed that there is a failure in diagnosis, since many lesions are asymptomatic and mistakenly treated as periodontal disease. Our patients were asymptomatic in $89 \%$ of the cases, while others could not identify their symptoms, which culminates in late diagnosis and consequent worsening of the disease, leading to long and resistant treatments. ${ }^{31}$

It is more common among females than males (with a prevalence of 2:1); and more common in white skin color, thus agreeing with the data collected in this study. ${ }^{31}$

Differential diagnosis should always be based on microscopic analysis of perilesional incisional biopsies. Histopathology shows subepithelial cleft with intact basement membrane with an abundant underlying inflammatory infiltrate. When it is not decisive, it may be necessary in some cases to use complementary direct immunofluorescence, which is characterized by linear deposition of $\operatorname{IgG}$, IgA, and $\mathrm{C} 3$ in the basement membrane region. ${ }^{32}$

There is no clear-cut protocol for treatment of MMP; however, oral hygiene must be rigorously evaluated to minimize worsening of the lesions, given the high incidence rate in the gingiva. The use of soft bristle toothbrushes and milder toothpaste are recommended. Antibacterial agents such as $0.12 \%$ chlorhexidine can be applied as a mouthwash twice a day to minimize discomfort caused by the injuries. ${ }^{33}$

The therapeutic strategy varies according to doctor's preference, patient's age, severity of disease, and disease location. Topical and/or systemic corticosteroids with or without immunosuppressive drugs, Dapsone, and Tetracycline are among the widely alternative treatment regimens. ${ }^{34}$

Among the topical medications, studies have reported good results in patients with oral lesions. However, it can be difficult to apply and maintain corticosteroids in extensive and deep gingival lesions. In addition, physiological movements of the oral cavity can displace the medication from its initial location, thus reducing the contact time between the drug and lesions. The use of trays with medication seems to be a solution to this problem, as it keeps the corticosteroid in the injured tissue and promotes an occlusive therapy. Using a study model, a silicone tray is made, where $1 \%$ hydrocortisone gel is applied with $0.5 \%$ lidocaine for 30 minutes (thrice a day) after oral hygiene. Corticosteroids of first choice are generally of low potency, because as these lesions have a chronic course, these drugs are indicated for prolonged treatments and have few adverse effects. ${ }^{35}$

Lupus erythematosus is a chronic inflammatory disease of unknown cause and characterized by presence of several autoantibodies and different clinical presentations. It is suggested that there are hereditary, immunological, hormonal and environmental factors associated with its etiopathogenesis, which may be linked with other autoimmune diseases. Classically, lupus erythematosus is subdivided into systemic and discoid forms. While SLE affects several organs and has an uncertain prognosis, discoid lupus erythematosus is a less serious condition and is limited to the skin and/or mucous surfaces. ${ }^{36}$ In our study, all patients had systemic form of the disease during in follow-up and treatment by dermatologists and rheumatologists. The prevalence of oral mucosa involvement in these patients is variable. According to some authors, oral lesions are present in $5 \%-54 \%$ of SLE cases and $3 \%-25 \%$ of patients with the discoid variant, with wide spectrum clinical presentation which varies between white plaques with central erythema surrounded by whitish streaks, honeycomb-like erythematous plaques or ulcerated lesions. ${ }^{9,36}$ They have a predilection for areas of cheek mucosa, soft palate, vermilion of lower lip, and tongue. ${ }^{36}$ In our collection, the clinical presentation was quite varied, thus showing prevalence by areas of cheek mucosa, gingiva, and tongue, both concomitantly and isolatively, not excluding the lips region, thus corroborating data in literature. The disease has a predilection for females in second and third decades of their life, which was also observed in our study, in which women represented $91 \%$ of SLE cases. However, regarding the age of diagnosis, approximately $20 \%$ of SLE cases are diagnosed in childhood or adolescence, with mean age between 11 and 12 years, making it the most common autoimmune disease in children, with progression to impairment of multiple organs in a short period of time. ${ }^{37}$ It was observed that $27 \%$ of patients followed by us were diagnosed early, that is, under the age of 19 years, which is 
in line with the numbers found in literature, thus evolving to more unfavorable prognosis.

The diagnosis is mainly based on immunohistochemical findings, given the high clinical and histopathological similarity with OLP. This diagnostic method is based on a quantitative difference in markings for CD4, CD8, and CD20 in a higher concentration of tryptase in the epithelium of oral lesions of lupus erythematosus and greater expression of CD1a in epithelia and conjunctive of OLP lesions. The thickness of the basement membrane in PAS was although more significant in lupus lesions, but did not constitute a defining criteria for differential diagnosis between oral lesions of these entities. ${ }^{38}$

The treatment of oral lesions is based on the use of topical corticosteroids ( $0.1 \%$ triamcinolone, as first choice). The use depends on severity of the symptoms and recurrence rate. In this case, corticosteroids of greater potency are indicated such as oral preparations based on Betamethasone or Clobetasol, or systemic drugs. Hydroxychloroquine combined with corticosteroids seem to be very effective, especially when there are systemic symptoms. It is essential that these patients are strictly monitored by dermatologists and rheumatologists, especially when diagnosed at earlier ages, due to the high rate of associated systemic comorbidities. ${ }^{37}$

Some reports demonstrate possible a link between SLE and periodontal disease. However, this information is still controversial. Good clinical practice recommends attention to oral health of patients with SLE, with periodic dental evaluation. In the event of identification of periodontal disease, treatment must be carried out, since it can positively influence the evolution of SLE, given its high inflammatory potential. ${ }^{39}$

$\mathrm{EM}$ is a rare mucocutaneous disease caused by hypersensitivity reaction triggered by viral infections, such as herpes simplex virus (HSV) and Mycoplasma pneumoniae, or drugs, which induce keratinocyte apoptosis. This disease was classified according to different variants such as the degree of mucosal involvement, as well as nature and location of skin lesions. ${ }^{40}$ Occasionally, EM may affect only oral cavity and when this occurs, it commonly affects the tongue, cheek, and labial mucosa, with lower frequency on the mouth floor and palate; and is characterized by erosive and vesicular lesions, which ruptures to cause ulcers of irregular and extremely painful surfaces. ${ }^{40}$

In this study, clinical presentations were disseminated through the oral mucosa as well as on the lips alone, in which all clinical cases are symptomatic, corroborating with the literature.

Regarding the prevalence of sex, literature reports a slight predominance in females. In contrast to our study, the distribution was homogeneous between genders. However, it is worth noting that we obtained only 4 cases of EM and the low number of this sample is significant with any statistical comparisons in literature.

It mainly affects young and healthy adults between the 3rd and 4 th decade of their life, but $20 \%$ of cases were reported in children. ${ }^{14}$ In regard to age, our distribution was equal among patients under 19 and over 20 years of age.

The diagnosis is based exclusively on clinical findings. There is no specific laboratory test for EM and histopathological exams are merely suggestive, thus demonstrating an intense inflammatory infiltrate, which is being performed for the exclusion and differential diagnosis of other vesiculobullous disorders. ${ }^{41}$ Anamnesis is essential for identification of the causal factor and performance is essential of the dental surgeon. In cases of suspected association with HSV, in which the information provided by anamnesis is insufficient, tests such as viral culture and cytopathology can be performed..$^{14,41}$

Treatment basically consists of controlling the symptoms and preventing the lesions from getting worse, as well as reducing the frequency and severity of recurrences. If drug induction is suspected, it should be suspended immediately. In cases of viral infections, systemic antiviral therapy is initiated. ${ }^{41}$

For the healing process of oral lesions, topical corticosteroids remain the therapy of choice and may be associated with anesthetic solutions. ${ }^{3,8}$

The use of low power lasers is an alternative widely used in dentistry and has significant benefits in the management of EM injuries. With the biomodulation process induced by the lights obtained in this modality, we observed epithelial healing in shorter intervals, which returned the patients to a faster quality of life. ${ }^{42}$

CUS is a rare mucocutaneous disorder with few cases reported in the literature, ${ }^{12}$ thus corroborating our study that presented only one case. Given the scarcity of information in literature, the exact prevalence of this disorder in is unknown. In a systematic review by Ferrisse et al (2019), in a total of 12 selected studies, cases of 32 patients were reported, with a significant predominance of females, white skin color and age from the fifth decade of life, in which only 2 were men, 11 also corroborating our study that showed the disease in a 63 -year-old white woman. The skin is rarely affected, with the oral mucosa being the main site of involvement and back of the tongue, cheek mucosa and gingiva being the most involved sites. The patients in this study presented extensive simultaneous involvement of the tongue, cheek mucosa and gingiva, thus corroborating information in literature, ${ }^{11,43}$ which reports clinical aspects such as erosive and/ulcerated lesions associated with reticular streaks, with symptoms of pain, burning and dryness of the oral mucosa. ${ }^{43}$

CUS most often presents clinical and histopathological aspects that is though not specific, but similar to OLP, OLR, MMP, IgA Linear Disease, PV, EM, or PYV, ${ }^{43}$ 
which are differentially diagnosed through direct immunofluorescence. It is believed that there are many more cases than those reported in literature and that many cases have not received proper correct diagnosis and have been wrongly treated. ${ }^{11}$ In contrast to other immunemediated disorders, CUS does not respond to corticosteroid therapy performed using Hydroxychloroquine. ${ }^{43}$

Pyoestomatitis vegetans is a rare inflammatory condition of unknown origin. The association with inflammatory bowel disease is well established, occurring in $70 \%$ of cases. ${ }^{44}$ Oral and skin lesions can precede the onset of intestinal involvement reported in $15 \%$ of cases. Ulcerative colitis is described in 53\% of cases and Crohn's disease in $11 \%{ }^{45}$ Given this information, careful gastroenterological and dermatological referral and follow-up of these patients is essential. The patient in question in this study was immediately referred to a dermatologist and gastroenterologist for having disseminated cutaneous lesions in the trunk, limbs, and scalp, as well as history of ulcerative colitis. This disorder is characterized as a disease that occurs in the third decade of life, with a female:male ratio of $3: 1 .^{44}$ The patient in this study was a man, 20 years of age, differing from what was reported in literature.

The oral mucosa is strongly affected and is characterized by exophytic, pustular lesions with an erythematous halo, a friable membrane that ruptures, giving rise to ulcerated areas. The most affected sites are the gingiva, soft palate, tonsils, and vestibular mucosa. ${ }^{13}$ Similarly, the patient in this study also presented widespread lesions in the oral mucosa, but without painful symptoms.

The diagnosis is based on histopathological findings, which reveal a pseudoepitheliomatous hyperplasia and presence of Munro microabscesses and eosinophilic spongiosis. Like PV, acantholysis and intraepithelial clefts can be visualized. A strong eosinophilic predominance leads some authors to suggest that this disease be classified within the pattern of eosinophilic dermatoses. Due to the histopathological similarity that is presented with $\mathrm{PV}$, diagnosis of some cases are difficult, thus direct and indirect immunofluorescences may be needed. ${ }^{45}$

Corticosteroid treatment is very effective. Unlike autoimmune disorders in which the recurrence of lesions is a major factor in etiology of the disease, in PV, the response to corticosteroids is effective and definitive. Topical and systemic medications can be used, depending on evolution of the disease. The prognosis is excellent and tends to follow the course of the underlying disease. Food supplementation also shows significant improvement of bad intestinal absorption in the active disease phase. ${ }^{45}$

EB differs from other disorders in that it has a hereditary character, as it is characterized by mutations in genes that form any of the structural proteins, thus interfering with the functional and structural integrity of the basement membrane zone. It covers three main forms: simple, junctional, and dystrophic recessive, with at least 20 different clinical phenotypes. ${ }^{14}$ In this study, we obtained data from 4 patients with EB, 3 of which were simple and 1 was dystrophic recessive. The diagnosis is always confirmed by histopathological examination, even if the clinical manifestations already suggest the condition. Mutation analysis can be considered, but they are not recommended as first line diagnosis because they are expensive and have little availability and great genetic heterogeneity. ${ }^{14}$

The first manifestations occur in areas of greater friction and trauma to the epithelial lining, with the age of diagnosis generally being in childhood or adolescence, which corroborates our study, in which most cases (75\%) were diagnosed before the age of 19 . In the oral mucosa, food can trigger the appearance of painful blisters and ulcers due to the simple act of chewing and swallowing, thus affecting all anatomical sites in a widespread manner. Even with the development of scars and hand contracting, EB patients have limitations regarding oral hygiene issues, which further promote an exacerbation of injuries due to accumulation of bacterial plaque. All the 4 patients in this study had lesions spreading through the mucosa and with painful symptoms.

So far, there is no specific therapy. Therapy is based on control of pain, infection, and exacerbation of injuries. Infected wounds deserve systemic antibiotic therapy. If the pain is mild or moderate, analgesics and non-steroidal anti-inflammatory drugs are prescribed. If intense, opioids and/or anxiolytics are administered. ${ }^{46}$ All patients severely affected have a nutritional deficit requiring iron replacement with or without erythropoietin. Ophthalmological and oral mucosa involvement should be evaluated together with the expertise of an ophthalmologist and the dentist. ${ }^{13}$

\section{Conclusion}

According to the results obtained in this study, we conclude that dermatological diseases affect the oral mucosa more frequent, with a significant impact on quality of life of the patients, given the high rates among young patients. Of the diseases reported in this study, pemphigus vulgaris was the most prevalent. Multiple presentations can be asymptomatic, thus delaying the diagnosis and worsening the condition and making the estimation of these diseases underreported. This information reinforces the need for strict monitoring by the dentist, given the great occurrence of oral manifestations prior to appearance of skin lesions in most of the data collected. Owing to the fact that they are disorders with no cure and/or variable prognosis, continuous monitoring by a multidisciplinary team is essential. 


\section{Acknowledgments}

The authors acknowledge the Oral Pathology Laboratory School of Dentistry - Rio de Janeiro State University; the Postgraduate Program in Pathology, School of Medicine - Fluminense Federal University; the BioNeo Pathologic
Anatomy and Citopathology Laboratory - Rio de Janeiro; the Pathology Laboratory of Pedro Ernesto University Hospital - State University of Rio de Janeiro; and the students from the Stomatology Service of the Piquet Carneiro Polyclinic - State University of Rio de Janeiro.

\section{References}

1. Lerner G, Jeremias P, Matthias T. The world incidence and prevalence of autoimmune diseases is increasing. Int J Celiac Diseas 2015; 3:151-5.

2. De Rossi SS, Ciarrocca K. Less common oral diseases of medical significance. J Calif Dent Assoc 2016; 44:571-6.

3. Mustafa MB, Porter SR, Smoller BR, Sitaru C. Oral mucosal manifestations of autoimmune skin diseases. Autoimmun Rev 2015; 14:930-51.

4. Porro AM, Seque CA, Ferreira MCC, Simões MM, Enokihara S. Pemphigus vulgaris. An bras dermatol 2019; 94:264-78.

5. DeAngelis LM, Cirillo N, McCullough MJ. The immunopathogenesis of oral lichen planus-is there a role for mucosal associated invariant $\mathrm{T}$ cell? J Oral Pathol Med 2019; 10:1-8.

6. Mostafa B, Ahmed E. Prevalence of oral lichen planus among a sample of the Egyptian population. J Clin Exp Dent 2015; 7:e7-e12.

7. Thete SG, Kulkarni M, Nikam AP, Mantri T, Umbare D, Satdive S, et al Oral manifestation in patients diagnosed with dermatological diseases. J Contemp Dent Pract 2017; 18:1153-8.

8. Saccucci M, DiCarlo G, Bossù M, Giovarruscio F, Salucci A, Polimeni A. Autoimmune diseases and their manifestations on oral cavity: diagnosis and clinical management. Journal of Immunology Report. J Immunol Res 2018; 2018:6061825.

9. Menzies S, O'Shea F, Galvin S, Wynne B. Oral manifestations of lupus. Ir J Med Sci 2018; 187:91-3.

10. Lerch M, Mainetti C, Terziroli Beretta-Piccoli B, Harr T. Current perspectives on erythema multiforme. Clin Rev Allergy Immunol 2018; 54:177-84.

11. Ferrisse TM, Travassos DC, Rocha AF, Massucato EM, Bufalino A. Chronic ulcerative stomatitis: a systematic review of the clinical and microscopic features. Med oral patol oral cir bucal 2019; 24:e698-703.

12. Pereira MS, Munerato MC. Oral Manifestations of inflammatory bowel diseases: two case reports. Clin Med Res 2016; 14:46-52.

13. Von der Lippe C, Diesen PS, Feragen KB. Living with a rare disorder: a systematic review of the qualitative literature. Mol Genet Genom Med 2017; 5:758-73.

14. Cizenski JD, Michel P, Watson IT, Frieder J, Wilder EG, Wright JM, et al.. "Spectrum of orocutaneous disease associations: immune-mediated conditions." J Am Acad Dermatol 2017; 77:795-806.

15. Vinall C, Stevens L, McArdle P. Pemphigus vulgaris: a multidisciplinary approach to management. BMJ Case Rep 2013; 2013:1-11.

16. Celere BS, Vernal S, Brochado MJF, Segura-Muñoz SI, Roselino AM. Geographical foci and epidemiological changes of pemphigus vulgaris in four decades in Southeastern Brazil. Int J Dermatol 2017; 56:1494-6.

17. Amagai M. Pemphigus. In: Bolognia JL, Schaffer JV, Cerroni L Dermatology. 4th ed. New York: Elsevier. 2018: 494-509.

18. Kridin K, Zelber-Sagi S, Khamaisi M, Cohen AD, Bergman R. Remarkable differences in the epidemiology of pemphigus among two ethnic populations in the same geographic region. J Am Acad Dermatol 2016; 75:925-30.

19. Arbabi M, Ghodsi Z, Mahdanian A, Noormohammadi N, Shalileh K, Darvish F, et al. Mental health in patients with pemphigus: an issue to worth consideration. Indian J Dermatol 2011; 56:541-5.

20. Yan L, Wang JM, Zeng K. Association between HLA-DRB1 and pemphigus vulgaris: a meta-analysis. Br J Dermatol 2012; 167:768-77.

21. Dal Prá KJ, Tristao SDSS, Franco JB, Matias DT, Carrillo CM, Peres MPSM, et al. Oral management of pemphigus vulgaris in the intensive care unit. Spec Care Dentist 2020; 30: 1-5.

22. Gupta S, Jawanda MK. Oral Lichen planus: an update on etiology, pathogenesis, clinical presentation, diagnosis and management. Indian J Dermatol 2015; 60:222-9.

23. Werneck JT, Miranda FBd, Silva Junior Jr A. Desafios na distinção de lesões de líquen plano oral e reação liquenóide. Rev Bras Odontol
2016; 73:247-52.

24. Suresh L, Neiders ME. Definitive and differential diagnosis of desquamative gingivitis through direct immunofluorescence studies. J Periodontol 2012; 83:1270-8.

25. Pippi R, Romeo U, Santoro M. Psychological disorders and oral lichen planus: matched case-control study and literature review. Oral Dis 2016; 22:226-34.

26. Teixeira-Souza T, Bombarda-Nunes FF, Leal LF, Pigatti FM. Chapter 3. Lichen planus: pathogenesis, diagnosis and treatments. Nova. In: Oral lichen planus: a review of different forms of treatment Medicine \& health; 54. 2020: 41-51.

27. Schiavo AL, Puca RV, Ruocco V, Ruocco E. Adjuvant drugs in autoimmune bullous diseases, efficacy versus safety: facts and controversies. Clin Dermatol $2010 ; 28: 337-43$

28. Ghahremanlo A, Boroumand N, Ghazvini K, Hashemy SI. Herbal medicine in oral lichen planus. Phytther Res 2019; 33:288-93.

29. Mostafa B, Zakaria M. Evaluation of combined topical ozone and steroid therapy in management of oral lichen planus. J Med Sci 2018; 6:879-84.

30. Carey B, Setterfield J. Mucous membrane pemphigoid and oral blistering diseases. Clin Exp Dermatol 2019; 44:732-9.

31. Hübner F, Recke A, Zillikens D, Linder R, Schmidt E. Prevalence and age distribution of pemphigus and pemphigoid diseases in Germany. J Invest Dermatol 2016; 136:2495-8.

32. Shimanovich I, Nitz JM, Zillikens D. Multiple and repeated sampling increases the sensitivity of direct immunofluorescence testing for the diagnosis of mucous membrane pemphigoid. J Am Acad Dermatol 2017; 77:700-705.e3.

33. Garcia-Pola MJ, Rodriguez-Lopez S, Fernánz-Vigil A, Bagán L, GarciaMartín LM. Oral hygiene instructions and professional control as a part of the treatment of desquamative gingivitis: systematic review. Med Oral Pathol Oral Cir Oral 2019; 24:136-44.

34. Kiran MS, Vidya S, Aswal GS, Kumar V, Rai V. Systemic and topical steroids in the management of oral mucosal lesions. J Pharm Bioallied Sci 2017; 9:S1-3. 35. Oliveira GMR, Pereira HSC, Silva-Júnior GO, Picciani BLS, Dias EP, Cantisano MH. Use of occlusive corticosteroid for the treatment of desquamative gengivitis: an effective option. Rev Bras Odontol 2013; 70:89-92.

36. Lourenço SV, de Carvalho FR, Boggio P, Sotto MN, Vilela MA, Rivitti EA, et al. Lupus erythematosus: clinical and histopathological study of oral manifestations and immunohistochemical profile of the inflammatory infiltrate. J Cutan Pathol 2007; 34:558-64.

37. Rodsaward P, Prueksrisakul T, Deekajorndech T, Edwards SW, Beresford MW, Chiewchengchol D. Oral ulcers in juvenile-onset systemic lupus erythematosus: a review of the literature. Am J Clin Dermatol 2017; 18:755-62.

38. Fava M. Diagnóstico diferencial de lesões orais de liquen plano e lupus eritematoso através de imunoistoquímica e PAS. Pontifícia Univ RIO Grande Sul Doutorado 2011.

39. Sete MRC, Figueredo CMdS, Sztajnbok F. Doença periodontal e lúpus eritematoso sistêmico. Rev Bras Reumatol 2016; 56:165-70.

40. Infen-Housz-Oro S, Ortonne N, Chosidow O. The diagnosis is in the rings. BMJ 2017; 359:j3817.

41. Sokumbi O, Wetter DA. Clinical features, diagnosis and treatment of erythema multiforme. A review of practicing dermatologist. Int J Dermatol 2012; 51:889-902.

42. Lins RDAU, Dantas EM, Lucena KCR, Catão MHCV, Granville-Garcia AF, Carvalho Neto LG. Efeitos bioestimulantes do laser de baixa potência no processo de reparo. An bras dermatol 2010; 85:849-55.

43. Islam MN, Cohen DM, Ojha J, Stewart CM, Katz J, Bhattacharyya I. Chronic 
ulcerative stomatitis: diagnostic and management challenges-four new cases and review of literature. Oral Surg Oral Med Oral Pathol Oral Radiol Endod 2007; 104:194-203.

44. Ko HC, Jung DS, Jwa SW, Cho HH, Kim BS, Kwon KS, et al. Two cases of pyodermatitis-pyostomatitis vegetans. J Dermatol 2009; 36:293-7.
45. Matias Fde A, Rosa DJF, Carvalho MTF, Castañon MCMN. Pyodermatitis-Pyoestomatitis vegetans: case report and review of medical literature. An bras dermatol 2011; 86 Supplement 1:S137-40.

46. Watterson G, Howard R, Goldman A. Peripheral opioids in inflammatory pain. Arch Dis Child 2004; 89:679-81.

\section{Mini Curriculum and Author's Contribution}

1. Thays Teixeira de Souza - DDS; MsC. Manuscript writing, manuscript review. ORCID: 0000-0002-5957-3239

2. Thiago Moreira Pêssoa - DDS; MsC. Manuscript review. ORCID: 0000-0002-4596-9478

3. Ruth Tramontani Ramos - DDS; PhD student. Manuscript review. ORCID: 0000-0001-8202-4855

4. Marilia Heffer Cantisano - DDS; PhD. Manuscript review. ORCID: 0000-0002-3121-295X

5. Geraldo Oliveira Silva-Júnior - DDS; PhD. Manuscript writing, manuscript review, statistical analysis and work supervisor. ORCID: 0000-0003-0987-2684

Submitted: 10/05/2020 / Accepted for publication: 12/14/2020

Corresponding author:

Thays Teixeira de Souza

E-mail: thaystsouza@gmail.com 